\title{
Introducing Context-Awareness and Adaptation in Telemedicine Systems
}

\author{
Charalampos Doukas, Ilias Maglogiannis, and Kostas Karpouzis
}

\begin{abstract}
Proper coding and transmission of medical and physiological data is a crucial issue for the effective deployment and performance of telemedicine services. This chapter presents a platform for performing proper medical content adaptation based on context awareness. Sensors are used in order to determine the status of a patient being monitored through a medical network. Additional contextual information regarding the patient's environment (e.g., location, data transmission device and underlying network conditions, etc.) is represented through an ontological knowledge base model. Rule-based evaluation determines proper content (i.e., biosignals, medical video and audio) coding and transmission of medical data, in order to optimize the telemedicine process. The paper discusses the design of the ontological model and provides an initial assessment.
\end{abstract}

\section{Introduction}

A number of telemedicine applications exist nowadays, providing remote medical action systems (e.g., remote surgery systems), patient remote telemonitoring facilities (e.g., homecare of chronic disease patients), and transmission of medical content for remote assessment ([[1]]-[5]). Such platforms have been proved significant tools for the optimization of patient treatment offering better possibilities for managing chronic care, controlling health delivery costs and increasing quality of life and quality of health services in underserved populations. Collaborative applications that allow the exchange of medical content (e.g., a patient health record) between medical experts for educational purposes or for assessment assistance are also considered of great significance ([6]-[8]). Due to the remote locations of the involved actuators, a network infrastructure (wired and/or wireless) is needed to enable the transmission of the medical data. The majority of the latter data is

\author{
Charalampos Doukas \\ University of the Aegean, Department of Information \& Communication Systems \\ Engineering, Greece \\ Ilias Maglogiannis \\ University of Central Greece, Department of Biomedical Informatics Lamia, Greece \\ Kostas Karpouzis \\ Image, Video and Multimedia Systems Lab, \\ National Technical University of Athens, Greece
}

M. Wallace et al. (eds.): Semantics in Adaptive and Personalized Services, SCI 279, pp. $163-185$. springerlink.com

(C) Springer-Verlag Berlin Heidelberg 2010 
usually medical images and/or medical video related to the patient. Thus, telemedicine systems cannot always perform in a successful and efficient manner; Issues, like large data volumes (e.g., video sequences or high quality medical images), unnecessary data transmission occurrence and limited network resources can cause inefficient usage of such systems ([9], [10]). In addition, wired and/ or wireless network infrastructures often fail to deliver the required quality of service (e.g., bandwidth requirements, minimum delay and jitter requirements) due to network congestion and/or limited network resources. Appropriate content coding techniques (e.g., video and image compression) have been introduced in order to assess such issues ([11]-[13]), however the latter are highly associated to specific content type and cannot be applied in general. Additionally, they do not consider the underlying network status for appropriate coding and still cannot resolve the case of unnecessary data transmission. Scalable coding and context-aware medical networks can overcome the aforementioned issues, through performing appropriate content adaptation.

The realization and integration of Semantic Medical Devices can allow:

- To develop solutions for the realization of smart hospitals.

- To provide mobile access to the patient's Electronic Health Record (EHR).

- To enable the medical devices to send parts of the EHR (i.e. measurement results), alerts, status information etc. to the PDA of the physician.

- To develop medical devices that inherently support the interoperability among each other.

- To enable medical devices to send alerts (i.e. SMS) to the handheld devices (i.e. mobile, pager) of the caregivers of the patients, if something goes wrong with the patient.

- To develop solutions that provides pervasive healthcare services (everywhere, anytime) to the patients, whether staying at home or mobile.

- To develop solutions for ambient assistant living for the elderly patients (i.e. medication reminders, cognitive assistance etc.)

In addition, policy and rule-based mechanisms can provide better adaptivity of medical networks: For example, there is a need to adapt the frequency of measurements on a sensor depending on the activity and clinical condition of the patient. This enables optimizing power consumption whilst ensuring that important episodes are not missed. Similarly, the use of variable thresholds for transmitting sensor readings reduces the need for communication and thus power consumption. Typically, sensor configuration may also change depending on the user's context, e.g., location, current activity and medical history. Physiological parameters such as heart rate thresholds then need to be configured and customized accordingly. Policy-based techniques have been used for over a decade in network and systems management in order to define how the system should adapt in response to events such as failures, changes of context or changes in requirements. By specifying the policies (i.e., what actions should be performed in response to an event) declaratively and separately from the implementation of the actions, it is possible to dynamically change the adaptation directives without changing the implementation 
or interrupting the functioning of the device. Thus, policy-based mechanisms provide feedback control over the system and a constrained form of programmability.

This chapter presents a context aware medical content adaptation platform that utilizes semantic representation of the content and the context. Using proper reasoning techniques, content adaptation is performed; medical image and video transmission only when determined necessary and encode the transmitted data properly according to the network availability and quality, the user preferences and the patient status. The framework's architecture is open and does not depend on the monitoring applications used, the underlying networks or any other issues regarding the telemedicine system used. The rest of the paper is organized as follows: Section 2 presents the notion of context awareness in telemedicine platforms as found in literature and Section 3 discusses design issues in context-aware medical networks. Section 4 provides information on how context awareness can be achieved, whereas Section 5 discusses context representation issues. Section 6 describes content adaptation techniques and Section 7 provides information regarding the reasoning scheme based on semantic rules for the content adaptation decision. Section 8 presents the proposed platform architecture and Section 9 concludes the chapter.

\section{Related Work}

Context-awareness has been around for more than six years and a lot has been written on this concept. There are several different applications and application frameworks for modeling and evaluating context but only a few in the domain of healthcare and telemedicine. JCAF [40] is built to operate using a network approach wherein different sensory, control and output devices are connected in a peer-to-peer fashion. Entities such as locations, persons or items have their own context, in which context items can be placed. Entities can request and set context items, and/or subscribe to context changes. An example usage described is the context-aware interactive hospital bed [40]. A touch screen computer attached to a patient's bed uses context, and adjusts its display on context changes, effectively interacting with the environment. Based on proximity, entities and users are identified and authenticated such that a different interface can be shown to surgeons, to nurses, or other personnel. The experimental setup is able to detect RFID chips on medicine trays, and match the retrieved information with known entities within the infrastructure, such that it is able to distinguish between several physical objects. Using the beds context and patient information, the bed is able to tell whether or not the medicines on the medicine tray are actually prescribed to the patient or are misplaced by a nurse, potentially becoming a health risk to the patient. As an alternative, [41] describes a service-based infrastructure. The chapter poses the position that "to greatly simplify the task of creating and maintaining context-aware systems, we should shift as much of the weight of context-aware computing onto network-accessible middleware infrastructures". Although the do not cover privacy in the application infrastructure they recognize that regarding sensory input "if it were processed in a context infrastructure, it is likely that the interactivity would be stilted due to network latency". Biegel and Cahill [42] describe a model 
designed for mobile context-aware applications based on ubiquitous computing. Their approach, called sentient object model, describes a network of sensors, actuators and services which run independently but interact in an ad hoc setup.

All the aforementioned works present general frameworks for medical context modeling and utilize the latter for the provision of specific health services. To our best knowledge there is no other framework in the literature that exploits context awareness for proper medical content adaptation in telemedicine.

\section{Design Issues in Context-Aware Medical Networks}

The goal of research into context-awareness in clinical work is to provide a conceptual and technical framework, which can help application programmers create context-aware clinical computer systems. Such a framework should enable the programmer to design, develop, and deploy application-specific context-awareness features that are required in specific usage settings, while it automatically supports aspects of context-awareness, which are common across applications. This approach is similar to other frameworks and toolkits supporting the development of context-aware application, like the Context Toolkit [43]. Requirements for context awareness systems and/or frameworks have been widely discussed and described (see e.g. [43], [44], [45], [46], [47]). Context aware medical applications introduce however additional special requirements; In a hospital there are a wide range of clinical computer systems in use, and new systems are installed and removed on a regular basis. Furthermore, many clinicians (typical research active doctors) build their own applications, such as quality databases supporting a specific clinical experiment. In order to make such applications context-aware there is a need for a stable infrastructure that can be accessed by these applications, and there is a need for a programming interface used by the developers of such applications. The basic design principle in a context-awareness framework for medical purposes is therefore to divide it into two parts. One part supports the deployment of 'context services', which are robust, scalable, flexible adaptable, extensible, etc. Such services run independently of the applications supplying or using context information. The other part enables developers of context-aware applications to represent, acquire, handle, store, and use context information.

Considering the aforementioned, the main design requirements for contextaware medical networks can be summarized into the following:

Distributed and Cooperating Services: Gathering and applying context information is often tied to specific spaces or environments dedicated to a specific purpose. For example, using a context-aware computer system to aid and guide a surgeon is highly dependent on accurate and detailed context information about things going on in the operating room. Therefore, a context-awareness infrastructure should be distributed and loosely coupled, while maintaining ways of cooperating in a peerto-peer fashion.

Security and Privacy: Clinical data about patients are important context data for clinical applications, and such data should be handled secure and its privacy respected. For example, the hospital bed uses information about the treatment of the 
patient as context information, enabling it to adjust itself to the patient. Hence, context data should be protected, subject to access control, and not revealed to unauthorized clients. Therefore, the context services should embed an access control mechanism. Furthermore, it is important to know the validity of clients delivering context data.

Lookup and Discovery: Context-aware clinical application will continuously enter and leave the hospital, e.g. running on mobile equipment or being deployed as new applications. Such clients should be able to locate and connect to relevant context services in the infrastructure. Services are therefore required to register at Lookup and Discovery services and reveal what they can do.

Extensibility: Clinical applications using new context information and acquisition methods will constantly be deployed in treatment facilities. Therefore, a contextawareness infrastructure should be extensible in several ways. First, it should be possible to deploy, modify, and remove context services. Second, the infrastructure should support evolvement of supported types of context by dynamically load context definitions, functionality, and acquisition mechanisms, like new context sensors.

\section{Enabling Context Awareness}

Context awareness refers to the ability of systems to react based on their environment. Devices and computer systems may have information about the circumstances under which they are able to operate and based on rules, or an intelligent stimulus, react accordingly. The term context-awareness in ubiquitous computing was introduced by Schilit [14], [15]. Context aware devices may also try to make assumptions about the user's current situation. Dey defines context as "any information that can be used to characterize the situation of entities." [16].

Three important aspects of context are: (1) where the individual is; (2) who the individual is with; and (3) what resources are nearby. Although location is a primary capability, location-aware does not necessarily capture things of interest that are mobile or changing. Context-aware in contrast is used more generally to include nearby people, devices, lighting, noise level, network availability, and even the social situation; e.g., whether you are with your family or a friend from school.

In the domain of patient remote care context awareness refers to detection of patient status and appropriate adaptation of the medical services according to the latter status and environmental conditions.

\subsection{Patient Status Awareness}

Patient status awareness can be achieved by continuously monitoring the patient state through collecting information either directly related to the individual's health (e.g., biosignals like heart rate, temperature, blood oximetry and others summarized in Table 1) or information that can be processed and indicate emergency cases (e.g., detection of fall events, call for help, etc.). 
A broad definition of a signal is a 'measurable indication or representation of an actual phenomenon', which in the field of biosignals, refers to observable facts or stimuli of biological systems or life forms. In order to extract and document the meaning or the cause of a signal, a physician may utilize simple examination procedures, such as measuring the temperature of a human body or have to resort to highly specialized and sometimes intrusive equipment, such as an endoscope. Following signal acquisition, physicians go on to a second step, that of interpreting its meaning, usually after some kind of signal enhancement or 'pre-processing', that separates the captured information from noise and prepares it for specialized processing, classification and decision support algorithms.

Table 1 Broadly used biosignals with corresponding metric ranges, number of sensors required and information rate.

\begin{tabular}{llll}
\hline $\begin{array}{l}\text { Biomedical Measurements } \\
\text { (Broadly Used Biosignals) }\end{array}$ & $\begin{array}{l}\text { Voltage range } \\
(\mathrm{V})\end{array}$ & $\begin{array}{l}\text { Number of sen- } \\
\text { sors }\end{array}$ & $\begin{array}{l}\text { Information rate } \\
(\mathrm{b} / \mathrm{s})\end{array}$ \\
\hline ECG & $0.5-4 \mathrm{~m}$ & $5-9$ & 15000 \\
Heart sound & Extremely small & $2-4$ & 120000 \\
Heart rate & $0.5-4 \mathrm{~m}$ & 2 & 600 \\
EEG & $2-200 \mu$ & 20 & 4200 \\
EMG & $0.1-5 \mathrm{~m}$ & $2+$ & 600000 \\
Respiratory rate & Small & 1 & 800 \\
Temperature of body & $0-100 \mathrm{~m}$ & $1+$ & 80 \\
\hline
\end{tabular}

Biosignals require a digitization step in order to be converted into a digital form. This process begins with acquiring the raw signal in its analog form, which is then fed into an analog-to-digital (A/D) converter. Since computers cannot handle or store continuous data, the first step of the conversion procedure is to produce a discrete-time series from the analog form of the raw signal. This step is known as 'sampling' and is meant to create a sequence of values sampled from the original analog signals at predefined intervals, which can faithfully reconstruct the initial signal waveform. The second step of the digitization process is quantization, which works on the temporally sampled values of the initial signal and produces a signal, which is both temporally and quantitatively discrete; this means that the initial values are converted and encoded according to properties such as bit allocation and value range. Essentially, quantization maps the sampled signal into a range of values that is both compact and efficient for algorithms to work with.

The latter information is usually collected by equipment installed on the patient or on his/her surrounding environment and is transmitted to monitoring units. Proper processing and classification follows in order to detect the patient status from the data. 


\subsection{Patient Data Collection and Transmission}

The data acquisition is usually performed either through sensor devices placed on user's body or monitoring devices at the user's environment. The first collect biosignals, sounds, and/or movement related data, whereas the latter capture and process audiovisual content and generate estimation for events like patient falling, abnormal movement, distress situations like fire, etc. [17], [18], [19]. Previous works [25], [26] present overviews of such system and a prototype platform for detecting fall incidents and distress situation based on user motion and sound data. Sensor devices illustrated in Figure 1 have been used for data collection and transmission to the monitoring unit.

Regarding communication, there are two main enabling technologies according to their topology: on-body (wearable) and off-body networks. Recent technological advances have made possible a new generation of small, powerful, mobile computing devices. An off-body network connects to other systems that the user does not wear or carry and it is based on a Wireless Local Area Network (WLAN) infrastructure, while an on-body or Wireless Personal Area Network (WPAN) connects the devices themselves; the computers, peripherals, sensors, and other subsystems and runs at ad hoc mode.

Table 2 Wireless connection technologies for telemedicine systems.

\begin{tabular}{|c|c|c|c|}
\hline Technology & Data rate & Range & Frequency \\
\hline IEEE $802.11 \mathrm{a}$ & $54 \mathrm{Mbps}$ & $150 \mathrm{~m}$ & $5 \mathrm{GHz}$ \\
\hline IEEE $802.11 \mathrm{~b}$ & $11 \mathrm{Mbps}$ & $150 \mathrm{~m}$ & $2.4 \mathrm{GHz}$ ISM \\
\hline Bluetooth (IEEE 802.15.1) & $721 \mathrm{Kbps}$ & $10 m-150 m$ & $2.4 \mathrm{GHz}$ ISM \\
\hline HiperLAN2 & $54 \mathrm{Mbps}$ & $150 \mathrm{~m}$ & $5 \mathrm{GHz}$ \\
\hline $\begin{array}{l}\text { HomeRF (Shared Wireless Access } \\
\text { Protocol, SWAP) }\end{array}$ & $\begin{array}{l}\text { 1.6 Mbps (10 Mbps } \\
\text { for Ver.2) }\end{array}$ & $50 \mathrm{~m}$ & $2.4 \mathrm{GHz}$ ISM \\
\hline DECT & $32 \mathrm{kbps}$ & $100 \mathrm{~m}$ & $1880-1900 \mathrm{MHz}$ \\
\hline PWT & $32 \mathrm{kbps}$ & $100 \mathrm{~m}$ & $1920-1930 \mathrm{MHz}$ \\
\hline $\begin{array}{l}\text { IEEE } 802.15 .3 \text { (high data rate } \\
\text { wireless personal area network) }\end{array}$ & 11-55 Mbps & $1 \mathrm{~m}-50 \mathrm{~m}$ & 2.4GHz ISM \\
\hline $\begin{array}{l}\text { IEEE } 802.16 \text { (Local and } \\
\text { Metropolitan Area Networks) }\end{array}$ & $120 \mathrm{Mbps}$ & City limits & $2-66 \mathrm{GHz}$ \\
\hline $\begin{array}{l}\text { IEEE } 802.15 .4 \text { (low data rate } \\
\text { wireless personal area network), } \\
\text { Zigbee }\end{array}$ & $\begin{array}{l}250 \mathrm{kbps}, 20 \mathrm{kbps} \\
40 \mathrm{kbps}\end{array}$ & $100 m-300 m$ & $\begin{array}{l}2.4 \mathrm{GHz} \text { ISM, } \\
868 \mathrm{MHz} \\
915 \mathrm{MHz} \text { ISM }\end{array}$ \\
\hline IrDA & 4Mbps (IrDA-1.1) & $2 \mathrm{~m}$ & $\begin{array}{l}\text { IR }(0.90 \text { micro- } \\
\text { meter })\end{array}$ \\
\hline
\end{tabular}


Telemedicine systems set high demanding requirements regarding energy, size, cost, mobility, connectivity and coverage. Varying size and cost constraints directly result in corresponding varying limits on the energy available, as well as on computing, storage and communication resources. Low power requirements are necessary also from safety considerations since such systems run near or inside the body.

Mobility is another major issue for pervasive e-health applications because of the nature of users and applications and the easiness of the connectivity to other available wireless networks. Both off-body and personal area networks must not have line-of-sight (LoS) requirements. The various communication modalities (see Table 2) can be used in different ways to construct an actual communication network. Two common forms are infrastructure-based networks and ad hoc networks. Mobile ad hoc networks represent complex systems that consist of wireless mobile nodes, which can freely and dynamically self-organize into arbitrary and temporary, "ad hoc" network topologies, allowing devices to seamlessly inter-network in areas with no pre-existing communication infrastructure or centralized administration. The effective range of the sensors attached to a sensor node defines the coverage area of a sensor node. With sparse coverage, only parts of the area of interest are covered by the sensor nodes. With dense coverage, the area of interest is completely (or almost completely) covered by sensors. The degree of coverage also influences information processing algorithms. High coverage is a key to robust systems and may be exploited to extend the network lifetime by switching redundant nodes to power-saving sleep mode.
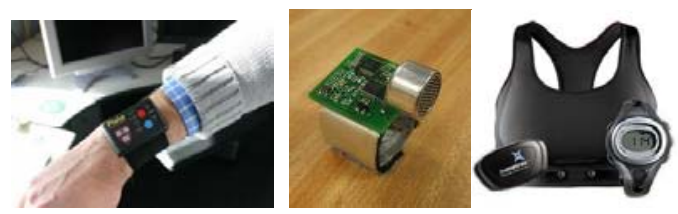

Fig. 1 Wearable medical sensor devices: (a) A 3-axis accelerometer on a wrist device enabling the acquisition of patient movement data [37], (b) A ring sensor for monitoring of blood oxygen saturation [38], (c) Wearable heart rate monitoring system by Numetrex [39].

\subsection{Medical Devices Access, Communication and Interoperability Issues}

The discovery and description of the medical devices must be semantic in order to discover appropriate medical devices to which one device wants to communicate. Thus, we suggest that the profiles of medical devices must be described by using existing ontologies, i.e. FIPA [17] or CC/PP [18], or by further specializing these ontologies for medical devices. The FIPA ontology specifies a frame-based structure to describe devices, and is intended to facilitate agent communication for purposes such as content adaptation. On the other hand, CC/PP is an RDF-based framework for describing software and hardware profiles of the devices, specifically to facilitate 
the decision making process of a server, on how to customize and transfer web content to a client device in a suitable format.

On the other hand, medical devices can also interoperate with existing legacy systems, being operated on different health standards, i.e. HL7, OpenEHR etc. As shown in Figure 2, a component with the name "Device Management Module" enriches the legacy systems with the capabilities of discovery and communication with external medical systems utilizing semantic annotations for devices and the retrieved context awareness. We suggest that this module should be developed for each of a particular health standard (i.e. HL7 v.2.3) compliant system in a language that can be executed on a number of platforms without its recompilation. The obvious choice for this purpose is Java, because the runtime environments to execute Java byte code exist for a number of platforms (software/hardware). Once this module has been developed for a particular health standard with the aforementioned capabilities, devices can easily discover this HIS/LIS and can query the functionalities that it provides and communicate with it seamlessly by understanding the semantic meanings of the functionalities that it offers.

\subsection{Semantic Medical Devices and Services}

We propose the use of Semantic Web Services (SWS) [14] to expose the functionalities of the medical devices as well as the functionalities of HISs/LISs, and to resolve the interoperability issues on each end. By exposing the various functionalities as Web Services and advertising them via SWS, medical devices can discover the services available in a hospital, laboratory or a clinic wherever they are physically present. Finally, the semantic descriptions of the Web Services provided by medical devices will automatically enable them to select, compose and execute the desired composite task.

Being a constituent part of the Ambient Intelligence, a medical device must have context-awareness capability, so that it could adapt itself to the rapidly changing situations. The various types of contextual information that can be used in the environment must be well defined so that different medical devices have a common understanding of the context. Also, there must be mechanisms for the medical device users to specify how different applications and services should behave in different contexts.

A proposed architecture for medical devices interoperability through semantics is illustrated in Figure 2. The Context Awareness Management (CAM) component manages the context awareness behavior of a medical device. It includes Context Manager (CM), which retrieves the contextual information from the subcomponents, i.e. Device Context, User Context, Security Context and the Physical Context. The device context provides information about the device (i.e. status, battery power etc.); the user context provides information about the user of the device (i.e. patient/health professional, personal prefers.); the physical context provides information about the present environment (i.e. hospital, clinic, laboratory, home etc.); and the security context provides information about the required and provided security level for a particular environment (i.e. a health professional must 


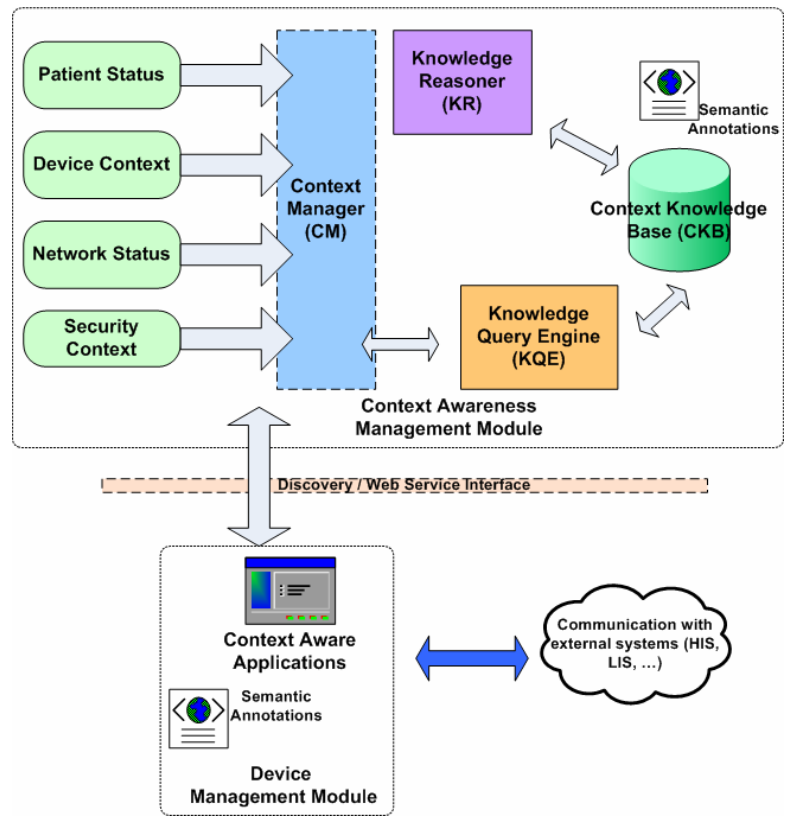

Fig. 2 Proposed Architecture for Medical Device Interoperability utilizing Context awareness and Semantic modules

provide his user identity (i.e. smart card, eToken) to send or receive patient's information from/on the device etc.). These sub-components provide basic contextual information in the form of context markups (i.e. an RDF graph), which support the $\mathrm{CM}$ not only to retrieve the contexts from Context Knowledge Base (CKB) through the Knowledge Query Engine (KQE), but also to infer higher-level contexts, with the help of Knowledge Reasoner (KR).

The CKB provides persistent knowledge storage, in the form of an extended context ontology for a particular environment (i.e. hospital, laboratory etc.) and the context markups that are given by the users or gathered from the basic context provider components (device context, physical context etc.). The CKB links the context ontology and markups in a single semantic model and provides interfaces for the KQE and the KR to manipulate correlated contexts. The KQE provides an abstract interface to the $\mathrm{CM}$ for extracting desired contexts from the CKB. To support expressive queries, any RDF Data Query Language can be used as context query language.

\subsection{Patient Location Technologies}

Positioning of individuals provides healthcare applications with the ability to offer services like supervision of elderly patients or those with mental illnesses who are ambulatory but restricted to a certain area. In addition, assisted care facilities can use network sensors and radiofrequency ID badges to alert staff members when 
patients leave a designated safety zone. Network or satellite positioning technology also can be used to quickly and accurately locate wireless subscribers in an emergency and communicate information about their location. Proximity information services can direct mobile users to a nearby healthcare facility. Locationbased health information services can help find people with matching blood types, organ donors, and so on. A more extensive list of location-based health services can be found in [21].

Positioning techniques can be implemented in two ways: Self-positioning and remote positioning. In the first approach, equipment that the user uses (e.g., a mobile terminal, or a tagging device) uses signals, transmitted by the gateways/antennas (which can be either terrestrial or satellite) to calculate its own position. More specifically, the positioning receiver makes the appropriate signal measurements from geographically distributed transmitters and uses these measurements. Technologies that can be used are satellite based (e.g., the Global Positioning System (GPS) and assisted-GPS), or terrestrial infrastructure-based (e.g., using the cell id of a subscribed mobile terminal).

The second technique is called remote positioning. In this case the individual can be located by measuring the signals traveling to and from a set of receivers. More specifically, the receivers, which can be installed at one or more locations, measure a signal originating from, or reflecting off, the object to be positioned. These signal measurements are used to determine the length and/or direction of the individual radio paths, and then the mobile terminal position is computed from geometric relationships; basically, a single measurement produces a straight-line locus from the remote receiver to the mobile phone. Another Angle Of Arrival (AOA) measurement will yield a second straight line, the intersection of the two lines giving the position fix for this system. Time delay can also be utilized: Since electromagnetic waves travel at a constant speed (speed of light) in free space, the distance between two points can be easily estimated by measuring the time delay of a radio wave transmitted between them. This method is well suited for satellite systems and is used universally by them. Popular applications that are based on the latter technique for tracking provision are the Ekahau Positioning Engine [22], MS RADAR [23] and Nibble [24]. More information regarding positioning techniques and systems can be found in [20].

\subsection{Data Processing and Classification}

The collected data contain information regarding the user's physiological status (in case of biosignals), potential distress situations (e.g., falls in case of movement data) and general information that can be correlated with the patient state. The data need further processing upon collection until the latter information can be acquired. Proper filtering might be required in order to remove irrelevant data like noise (e.g., in case of movement or sound data). In some cases patient state can be determined by applying simple value thresholds (e.g., in case of body temperature or heart rate) but in cases motion detection and interpretation advanced data classification techniques might be required. In [27] an overview of classification algorithms is 
presented that can be applied on movement and sound data collected by on-body sensors for patient fall event detection.

\subsection{User Environment Context Awareness}

Apart from determining the patient status, context aware medical treatment and monitoring systems must incorporate information related to user's environment. More specifically:

User's indoor or outdoor location can be determined by external devices (i.e. GPS, mobile or WLAN phones) and facilitate the process of ambulatory dispatching in case of emergency events. Based on location, proper proactive or reactive data transmission may also be performed. Information regarding the communication equipment used (e.g., laptop computer, mobile phone or PDA) can facilitate the content adaptation in case of video communication.

Transmission capabilities of the underlying networking infrastructures (e.g., network interface type used, allocated bandwidth, real time network traffic information, etc.) can affect the communication and thus facilitate the determination of proper content adaptation like application of compression schemes.

More information regarding context-aware medical networks and telemedicine services can be found at [28].

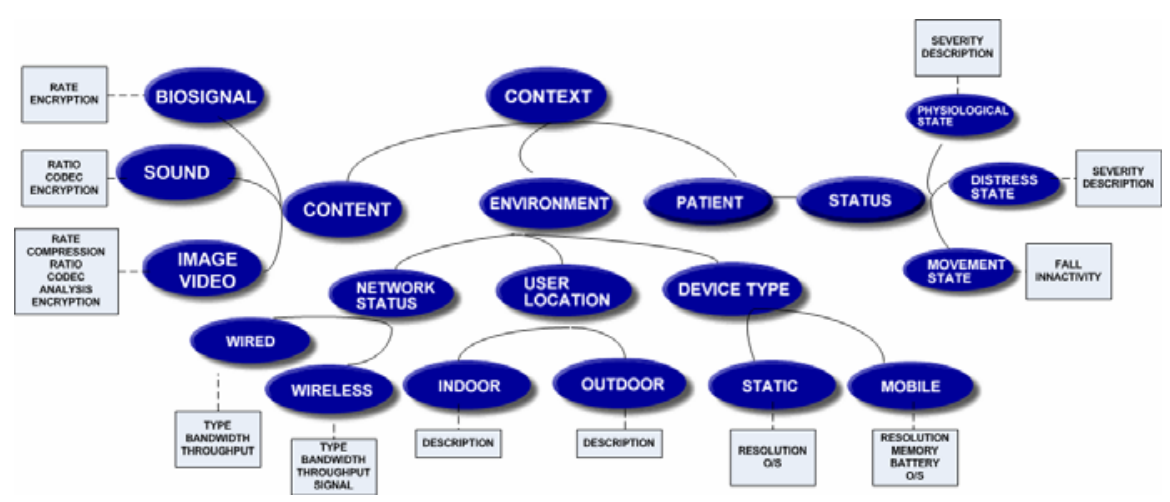

Fig. 3 Illustration of the semantic representation of the context aware data adaptation system using an ontological structure. Major component and actuator classes are illustrated among with most important features for each class.

\section{Context Semantic Representation}

In order to semantically represent the context aware system and the content adaptation the ontology illustrated in Figure 3 has been developed. Both the patientrelated context and content have been modeled. More specifically: regarding the medical content, a representative class with three subclasses has been created. Each subclass represents image and video medical data, audio data and biosignals 
respectively. Most important features for the proper content adaptation are the transmission data rate, type of encryption used (e.g., PKI [29], simple symmetric, or none), compression ratio (in case of scalable compression), codec used (e.g., H.264 for video, JPEG2000 for images and ITU G.723 for audio), and analysis (specifically for images and video according to the network status and the presentation device). The patient status is characterized according to physiological state, distress state (i.e. more generic from the latter containing status indications based on vocal and sound analysis), and movement state (e.g., detection of falls or long periods of inactivity). The basic attributes for the aforementioned states are the severity of the status (e.g., numerical representation of the emergency severity level), description of the incident and indication of fall or long inactivity status. A patient environment-related class has also been developed for representing the status of the underlying network infrastructures, the user location and the device types that are used for data collection, transmission. Concerning the network status, wired or wireless interfaces can be used. For both interfaces, the type of the medium, the total available bandwidth and the current throughput can affect the data transmission and thus content adaptation, whereas in the case of wireless interface the received signal strength might also be an important factor for the content adaptation. User location has been categorized into indoor and outdoor with a simple description as a respective attribute. Finally, the class "Device Type" refers to the transmission device the patient/user operates for communicating with the treatment/monitoring units. In case of static devices (e.g. PCs) the operating system and the screen resolution might determine content like the video analysis, and frame rate, whereas in case of mobile devices (e.g., mobile phones, PDAs, etc.) memory and power resources can also affect the transmission and presentation of the medical content respectively.

The ontological model has been developed within the Protégé [34] semantic framework using the Ontology Web Language (OWL). The main advantages of the semantic representation of the context aware adaptive system can be summarized into the following:

- Flexibility to modify and extend the contextual scheme by adding more classes. In case the parameters that define the context of the patient (e.g.., status, environment, location, etc.) need to be modified, the ontological model can be altered without invoking modifications to the implementation modules or the architecture of the platform.

- Better and more flexible evaluation of the context facilitating the decisions for the medical content adaptation. Using advanced semantic rule evaluation techniques (to be discussed in Section 5) content adaptation decisions can be made according to a plethora of contextual parameters. The rules can be updated and extended without any need for system platform software modifications.

Additionally, ontologies are explicit because define the concepts, properties, relationships, functions, axioms and constraints that compose the contextual model. They are formal because they are machine readable and interpreted. 


\section{Content Adaptation}

Content adaptation refers to proper medical data coding and proactive or reactive transmission for achieving better utilization of network and system resources during the monitoring and treatment process. The most demanding data in terms of network and system resources for transmission and processing are the medical and audiovisual data. Additionally, content adaptation can also include different data encryption schemes that can be applied according to data sensitivity and severity of an emergency incident.

\subsection{Image and Video/Audio Coding}

The coding of medical image and audiovisual data refers to data compression. According to the patient status and underlying network interfaces and conditions, several compression schemes can be applied; for instance, uncompressed data can be transmitted in case of a fast wired network connection, whereas higher compression schemes can be applied when using wireless connections with lower data rate availability. In case of visual assessment it might be important to maintain particular parts of the image/video of visual context at higher quality and increase the compression on less diagnostic important regions. Examples of special region of interest (ROI) coding with scalable compression can be found at [30], [31] for both medical image and video data.

\subsection{Adapted Data Security Policies}

The medical context as presented in previous sections contains sensitive information regarding the patient status, location and context of the surrounding environment. Therefore, several security issues are introduced and must be considered by context-aware medical networks:

- Maintaining information privacy, i.e. to prevent any disclosure of information directly related to the individual to a service or application without the user's prior approval or knowledge.

- Maintaining context privacy, i.e. to prevent any disclosure of information related to the context in which the user is using the service (for example her current device parameters) and from which indirect information for the user could be extracted.

- Maintaining location privacy of the user, i.e. to deny an attacker the knowledge of a device's current and past location and preventing linkability.

- Preserving anonymity of the users' identifiable parameters for distinct scenarios, i.e. preserving their "state of being not identifiable within a set of subjects". 
Proper solutions for resolving the latter issues can be:

- Mechanisms for protecting any type of sensitive information which the user considers private and for any level of granularity; the user decides how to protect her sensitive information and anonymity, and location privacy. Data abstractions over all types of low-level sensitive data are part of the mechanism and they are processed first allowing faster filtering and default setting;

- To help for the personalization, for hiding all the complexity of the system from the user, for delegating privacy decisions from the user to her device, descriptive profiles of user, user roles, scenarios, context are used.

- Rule-based access over the private data helps to delegate the decisions to the device and to take actions concerning the correct providing privacy parameters to the services

- Any time when the context attributes change, the privacy protection mechanisms evaluates the overall privacy status and acts accordingly based on the predefined rules

In the presented platform several data encryption schemes can be applied for providing medical content privacy, confidentiality, non repudiation and encryption. According to the sensitivity of the data and the severity of the case, simple symmetrical encryption schemes [32] to more complex public key infrastructures can be applied [33]. The platform decides according to specific context parameters, which data encryption methodology will be utilized prior to transmission. For instance in case of an emergency incident in an area where only low-bandwidth networks are available, the platform skips the encryption process.

\subsection{Reactive Data Transmission}

Unnecessary transmission of medical data or monitoring data (e.g., video from user's environment) can be avoided by using reactive data transmission. In case of normal patient state, data related to the patient context and status (e.g., visual data and biosignals) can be transmitted to monitoring units proactively in specified time intervals. In case of a detected distress situation, reactive transmission can begin. More information on data transmission based on context awareness can be found in [28].

\section{Content Adaptation Based on Semantic Rules Evaluation}

In order to perform the appropriate medical content adaptation that has been discussed in the previous sections, several semantic rules have been defined. These rules concern features of the ontological class that represents the context aware model semantically. By performing proper evaluation of the latter, decision regarding the content adaptation can be made. 
The creation of semantic rules required the description of the latter through abstract semantic languages like the Semantic Web Rule Language (SWRL) [35]. The syntax for SWRL abstracts from any exchange syntax for OWL [48] and thus facilitates access to and evaluation of the language. An OWL ontology in the abstract syntax contains a sequence of axioms and facts. Axioms may be of various kinds, e.g., subClass axioms and equivalentClass axioms. It is proposed to extend this with rule axioms.

$$
\text { axiom : : = rule }
$$

A rule axiom consists of an antecedent (body) and a consequent (head), each of which consists of a (posibly empty) set of atoms. A rule axiom can also be assigned a URI reference, which could serve to identify the rule.

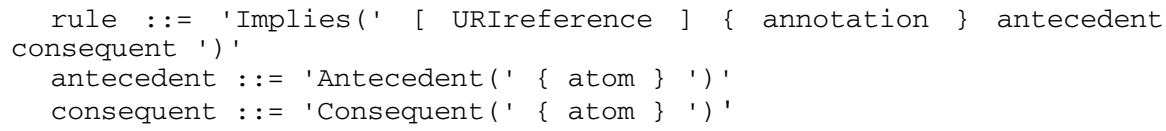

Informally, a rule may be read as meaning that if the antecedent holds (is "true"), then the consequent must also hold. An empty antecedent is treated as trivially holding (true), and an empty consequent is treated as trivially not holding (false). Rules with an empty antecedent can thus be used to provide unconditional facts; however such unconditional facts are better stated in OWL itself, i.e., without the use of the rule construct. Non-empty antecedents and consequents hold if all of their constituent atoms hold, i.e., they are treated as conjunctions of their atoms. Rules with conjunctive consequents could easily be transformed into multiple rules each with an atomic consequent.

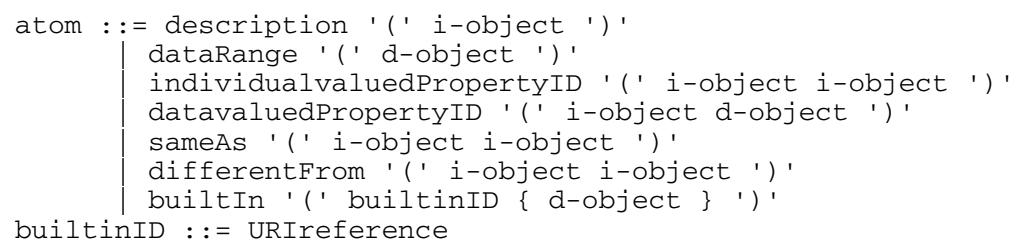

Atoms can be of the form $\mathrm{C}(\mathrm{x}), \mathrm{P}(\mathrm{x}, \mathrm{y})$, sameAs(x,y) differentFrom(x,y), or builtIn $(\mathrm{r}, \mathrm{x}, \ldots)$ where $\mathrm{C}$ is an OWL description or data range, $\mathrm{P}$ is an OWL property, $\mathrm{r}$ is a built-in relation, $\mathrm{x}$ and $\mathrm{y}$ are either variables, OWL individuals or OWL data values, as appropriate. Atoms may refer to individuals, data literals, individual variables or data variables. Variables are treated as universally quantified, with their scope limited to a given rule. As usual, only variables that occur in the antecedent of a rule may occur in the consequent.

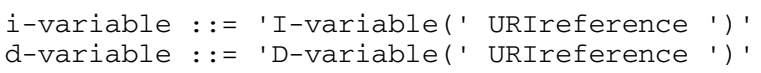

While this abstract syntax is consistent with the OWL specification, and is useful for defining XML and RDF serialisations, it is rather verbose and not particularly 
easy to read. Often a relatively informal "human readable" form is used similar to that used in many published works on rules.

In this syntax variables are indicated using the standard convention of prefixing them with a question mark (e.g.,?x). Using this syntax, a rule asserting that the composition of parent and brother properties implies the uncle property would be written:

parent (?x, ?y) -> brother (?y, ?z) -> uncle(?x, ?z)

Within this context, the SWRL Factory [34] mechanism and an integrated Jess rule engine [36] using the Protégé tool have been utilized. Jess provides both an interactive command line interface and a Java-based API to its rule engine. This engine can be embedded in Java applications and provides a flexible two-way runtime communication between Jess rules and Java. The Jess system consists of a rule base, a fact base, and an execution engine.

Two indicative sample SWRL rules follow that can be used within the presented framework in order to facilitate the decision on the content adaptation based on patient's context parameters:

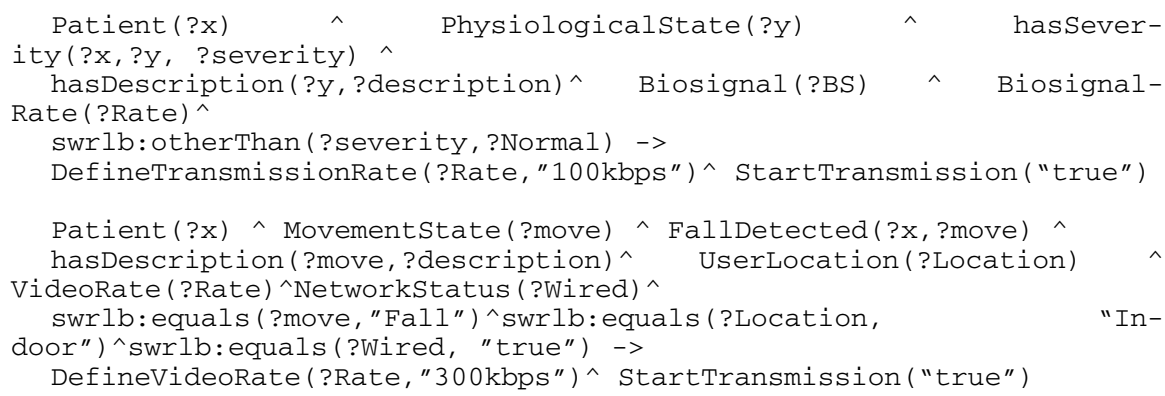

The first rule examines the physiological state of the patient as characterized by the status awareness modules in terms of status severity. If the latter is considered to be other than "Normal" then transmission of the collected biosignals to the monitoring units begins at a specific data rate. The second rule is more advanced and takes into consideration potential indication of a fall event, the location of the user and the network status. According to the rule, video transmission of the patient's premises will begin in case a fall has been detected. High transmission rate will be used if the user is located indoor and a wired network infrastructure is used.

\section{Proposed Architecture Scheme}

This Section presents the proposed architecture scheme that incorporates modules that feature the discussed aspects of context awareness and medical content adaptation. The interconnection and communication of the different components can be illustrated as five different application layers (see Figure 4). Initial data acquisition from the sensor and monitoring devices is followed by proper processing for feature extraction. The context awareness is performed by classifying the generated features 
and utilizing semantic evaluation of the latter. Application of semantic rules facilitates the determination of patient status and detection of emergency events.

According to the detected patient status and additional contextual information regarding the patient's environment and underlying network conditions, proper content adaptation to the medical data is performed. The content related to incident is coded (i.e. compressed and encrypted) accordingly and transmitted to the monitoring units.

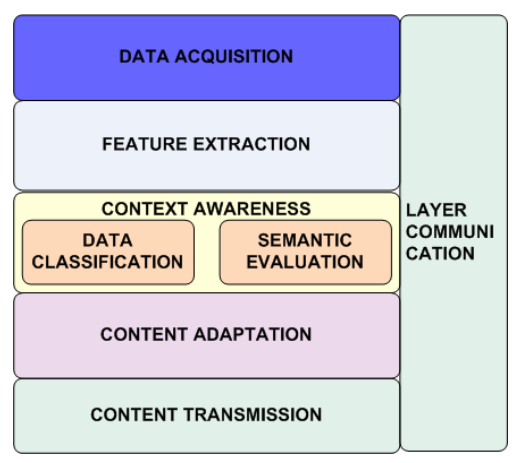

Fig. 4 Illustration of the incorporated application layers for context awareness and content adaptation and transmission.

Figure 5 illustrates a proposed architecture scheme for interconnected all the involved components for enabling context awareness and proper content coding.

The provision of the contextual data (i.e., estimated patient status based on rules evaluation, medical data and other context data) can be performed either through appropriate web-based and application interfaces or through creating appropriate Web Services, as discussed in the following paragraph.

\subsection{Context Information Provision through Web Services}

Web Services are emerging as a promising technology to build distributed applications. It is an implementation of Service Oriented Architecture (SOA) that supports the concept of loosely-coupled, open-standard, language - and platform-independent systems. Web Services are accessed through the HTTP/HTTPS protocols and utilize XML (eXtendible Markup Language) for data exchange. This in turn implies that Web Services are independent of platform, programming language, tool and network infrastructure. Services can be assembled and composed in such a way to foster the reuse of existing back-end infrastructure. The basic SOA includes three service components: provider, requester and registry. WSDL (Web Service Description Language) is commonly defined by the service provider for invoking the service. SOAP (Simple Object Access Protocol) is adopted as message transfer protocol between requester and provider and UDDI (the Universal Description, Discovery and Integration) is used for service registration and discovery. 
The typical scenario illustrated in Figure 6 is based on publishing (WSDL reference), searching for a service and binding to a service provider. The XML messaging between service consumer and provider exploits the SOAP protocol. SOAP provides automatic marshalling/unmarshalling of the arguments, like Remote Procedure Call (RPC).

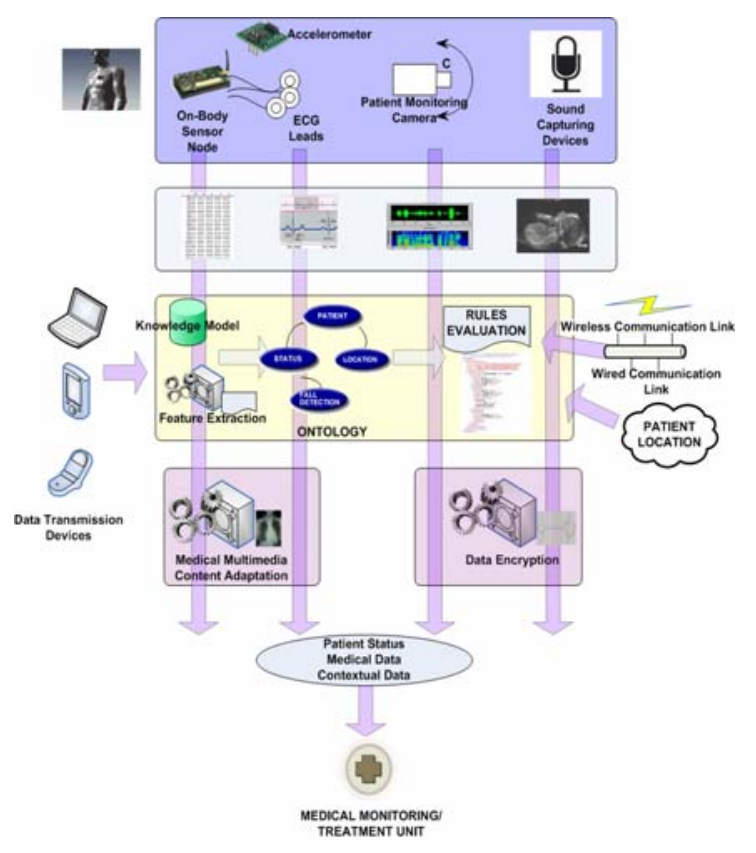

Fig. 5 The proposed architecture that incorporates modules and components for proper medical content adaptation based on context awareness.

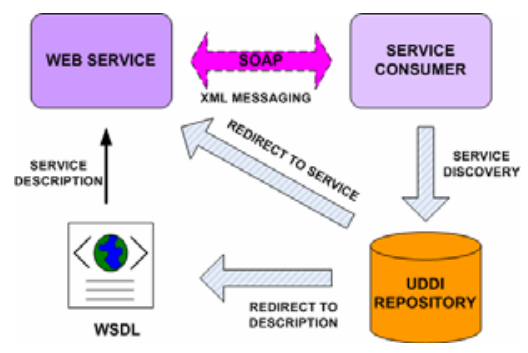

Fig. 6 General Web Services component framework

Web services provide several technological and business benefits, a few of which include application and data integration, versatility, code re-use and cost savings. The inherent interoperability that comes with using vendor, platform, and language independent XML technologies and the ubiquitous HTTP as a transport 
mean that any application can communicate with any other application using Web services. Web services are also versatile by design. They can be accessed by humans via a Web-based client interface, or they can be accessed by other applications and other Web services. Code re-use is another positive side-effect of Web services' interoperability and flexibility. One service might be utilized by several clients, all of which employ the operations provided to fulfill different business objectives.

In order to provide direct and efficient access to the contextual information generated by the platform, a Web Service module has been developed. The latter can expose specific functionality to developers for creating external client applications that can monitor the acquired biosignals of the platform, get information regarding the context of the patient and perform content adaptation based on rules evaluation. Figure 7 illustrates a sample WSDL definition for the developed Web Service. Two functions are described that concern the status of the patient and the parameters that describe the adaptation of the medical content based on the rules evaluation of the platform.

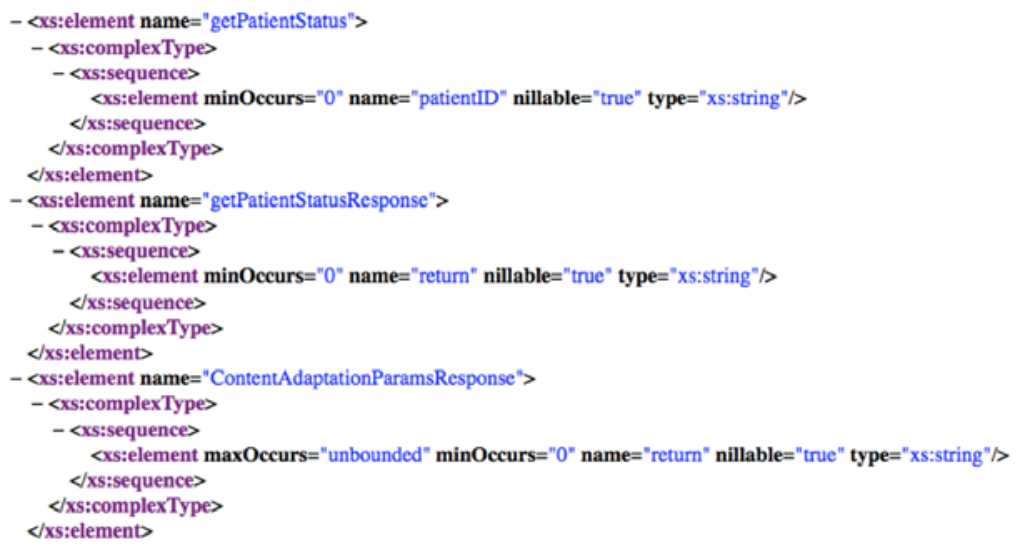

Fig. 7 WSDL sample description for the provided Web Services. "getPatientstatus" and "ContentAdaptationParams" refer to functions that provide information regarding the patient's state and the medical content adaptation parameters as indicated by the proposed framework.

\section{Conclusions}

A context-aware medical content adaptation platform has been presented. The platform utilizes sensor data for determining the patient status and takes into account additional contextual information like underlying network conditions, and data transmission devices. A semantic representation for the patient context has been developed and appropriate rule-based system is used in order to perform proper medical content adaptation according to the context, facilitating and improving the diagnosis and treatment process. In addition, a Web Service module provides access to information related to the context of the patient and the medical content adaptation. 
Future work might include the deployment of the proposed platform in a real remote treatment and monitoring environment for assessing the actual contribution of context awareness and content adaptation to the remote medical care process.

\section{References}

1. Lin, J.C.: Applying telecommunication technology to health care delivery. IEEE Engineering in Medicine and Biology Magazine 4, 28-31 (1999)

2. Pavlopoulos, S., Kyriacou, E., Berler, A., Dembeyiotis, S., Koutsouris, D.: A novel emergency telemedicine system based on wireless communication technologyAMBULANCE. IEEE Transactions on Information Technology in Biomedicine 4, 261-267 (1998)

3. Deb, S., Ghoshal, S., Malepati, V.N., Kleinman, D.L.: Tele-diagnosis: remote monitoring of large-scale systems. In: Proc. of IEEE Aerospace Conference, pp. 31-42 (2001)

4. Choi, Y.B., Krause, J.S.H., Seo, C.K., Chung, E.K.: Telemedicine in the USA: standardization through information management and technical applications. IEEE Communications Magazine 44, 41-48 (2006)

5. Pattichis, C.S., Kyriacou, E., Voskarides, S., Pattichis, M.S., Istepanian, R., Schizas, C.N.: Wireless telemedicine systems: an overview. IEEE Antennas and Propagation Magazine 44, 143-153 (2002)

6. Aakay, M., Marsic, I., Medl, A., Bu, G.: A system for medical consultation and education using multimodal human/machine communication. IEEE Transactions on Information Technology in Biomedicine 2(4), 282-291 (1998)

7. Zhou, J., Shen, X., Georganas, N.D.: Haptic tele-surgery simulation. In: Proc. of the 3rd IEEE International Workshop on Haptic, Audio and Visual Environments and their Applications, pp. 99-104 (2004)

8. Fontelo, P., DiNino, E., Johansen, K., Khan, A., Ackerman, M.: Virtual Microscopy: Potential Applications in Medical Education and Telemedicine in Countries with Developing Economies. In: Proc. of the 38th Annual Hawaii International Conference on System Sciences, p. 153 (2005)

9. Lage, A.-L., Martins, J., Oliveira, J., Cunha, W.: A quality of service approach for managing tele-medicine multimedia applications requirements. In: Proc. of IEEE Workshop on IP Operations and Management, pp. 186-190 (2004)

10. LeRouge, C., Garfield, M.J., Hevner, A.R.: Quality attributes in telemedicine video conferencing. In: Proc. of 35th Annual Hawaii International Conference on System Sciences, pp. 2050-2059 (2002)

11. Yu, H., Lin, Z., Pan, F.: Applications and improvement of H.264 in medical video compression. IEEE Transactions on Circuits and Systems 52(12), 2707-2716 (2005)

12. Bernabe, G., Gonzalez, J., Garcia, J.M., Duato, J.: A new lossy 3-D wavelet transform for high-quality compression of medical video. In: Proc. of IEEE EMBS International Conference on Information Technology Applications in Biomedicine, pp. 226-231 (2000)

13. Doukas, C.N., Maglogiannis, I., Kormentzas, G.: Medical Image Compression using Wavelet Transform on Mobile Devices with ROI coding support. In: Proc. of the 27th Annual International Conference of the IEEE EMBS, Shanghai, China,

14. Schilit, B., Adams, N., Want, R.: Context-aware computing applications. In: IEEE Workshop on Mobile Computing Systems and Applications (WMCSA 1994), Santa Cruz, CA, US, pp. 89-101 (1994) 
15. Schilit, B.N., Theimer, M.M.: Disseminating Active Map Information to Mobile Hosts. IEEE Network 8(5), 22-32 (1994)

16. Dey, A.K.: Understanding and Using Context. Personal Ubiquitous Computing 5(1), 4-7 (2001)

17. Wang, S., Yang, J., Chen, N., Chen, X., Zhang, Q.: Human activity recognition with user-free accelerometers in the sensor networks. In: Proc. of International Conference on Neural Networks and Brain, pp. 1212-1217 (2005)

18. Miaou, S.G., Sung, P.-H., Huang, C.-Y.: A Customized Human Fall Detection System Using Omni-Camera Images and Personal Information. In: Proc. of 1st Transdisciplinary conference on Distributed Diagnosis and Home Healthcare, pp. 39-42 (2006)

19. Istrate, D., Castelli, E., Vacher, M., Besacier, L., Serignat, J.F.: Information extraction from sound for medical telemonitoring. IEEE Transaction on Information Theory in Biomedicine 2(10), 264-274 (2006)

20. Zeimpekis, V., Giaglis, G.M., Lekakos, G.: A taxonomy of indoor and outdoor positioning techniques for mobile location services. ACM SIGecom Exchanges 3(4), 19-27 (2003)

21. Shih-wei, L., Shao-you, C., Yung-jen, H.J., Polly, H., Chuang-wen, Y.: Emergency Care Management with Location-Aware Services. In: Pervasive Health Conference and Workshops, pp. 1-6 (2006)

22. Ekahau LBS, http: / / www . ekahau. com (accessed on September 26, 2005)

23. Bahl, P., Padmanabhan, V.N.: RADAR: An In-Building RF-based User Location and Tracking System. In: INFOCOM, pp. 775-784. IEEE Press, Los Alamitos (2000)

24. Castro, P., Chiu, P., Kremenek, T., Muntz, R.: A Probabilistic Room Location Service for Wireless Networked Environments. In: Abowd, G.D., Brumitt, B., Shafer, S. (eds.) UbiComp 2001. LNCS, vol. 2201, pp. 18-34. Springer, Heidelberg (2001)

25. Doukas, C., Maglogiannis, I.: Enabling Human Status Awareness in Assistive Environments based on Advanced Sound and Motion Data Classification. Presented at The 1st ACM International Conference on PErvasive Technologies Related to Assistive Environments (PETRAE), Athens, Greece, July 16-19 (2008)

26. Doukas, C., Maglogiannis, I.: Advanced Patient or Elder Fall Detection based on Movement and Sound Data. Presented at 2nd International Conference on Pervasive Computing Technologies for Healthcare (2008)

27. Doukas, C., Maglogiannis, I.: Human Distress Sound Analysis and Characterization using Advanced Classification Techniques. Presented at 5th Hellenic Conference on Artificial Intelligence, Syros, Greece, October 2-4 (2008)

28. Doukas, C., Maglogiannis, I., Kormentzas, G.: Advanced Telemedicine Services through Context-aware Medical Networks. In: Proceedings of the IEEE EMBS cosponsored International Special Topic Conference on Information Technology in Biomedicine (ITAB 2006), Ioannina-Epirus, Greece, October 26-28 (2006)

29. Public Key Infrastructure, online information, http://www. ietf.org/html.charters/pkix-charter.html

30. Maglogiannis, Doukas, C., Kormentzas, G., Pliakas, T.: Optimized Mobile Access to DICOM Images using Wavelet compression with ROI coding support. To appear in IEEE Transactions on Information Technology in Biomedicine

31. Doukas, C., Maglogiannis, I.: Adaptive Transmission of Medical Image and Video using Scalable Coding and Context-aware Wireless Medical Networks. EURASIP Journal on Wireless Communications and Networking, Article ID 428397 2008, 12 (2008)

32. Makris, L., Argiriou, N., Strintzis, M.G.: Network and data security design for telemedicine applications. Informatics for Health and Social Care 22(2), 133-142 (1997) 
33. Bao, S.-D., Shen, L.-F., Zhang, Y.-T.: A novel key distribution of body area networks for telemedicine. In: Proc. of 2004 IEEE International Workshop on Biomedical Circuits and Systems, pp. 1-17-20a (2004)

34. Protégé Ontology Editor and Knowledge Base Framework, more information, http: / / protege.stanford.edu/

35. The Semantic Web Rule Language definition, http: / / www .w3 . org/Submission/SWRL/

36. The Jess Rule Engine, http: / /www. jessrules.com/jess/index.shtml

37. Malan, D., Fulford-Jones, T., Welsh, M., Moulton, S.: CodeBlue: An Ad Hoc Sensor Network Infrastructure for Emergency Medical Care. In: International Workshop on Wearable and Implantable Body Sensor Networks (2004)

38. Rhee, S., Yang, B.-H., Chang, K., Asada, H.H.: The Ring Sensor: a New Ambulatory Wearable Sensor for Twenty-Four Hour Patient Monitoring. In: Proceedings of the 20th Annual International Conference of the IEEE Engineering in Medicine and Biology Society, vol. 20(4), pp. 1906-1909 (1998)

39. Numetrex cardio shirt, http://www. numetrex.com/about/cardio-shirt

40. Bardram, J.E.: The Java Context Awareness Framework (JCAF): A Service Infrastructure and Programming Framework for Context-Aware Applications (2005)

41. Hong, J., Landay, J.: An Infrastructure Approach to Context-Aware Computing (2001)

42. Biegel, G., Cahill, V.: A Framework for Developing Mobile, Context-aware Applications (2004)

43. Dey, A., Abowd, G.D., Salber, D.: A conceptual framework and a toolkit for supporting the rapid prototyping of context-aware applications. Human-Computer Interaction 16, 97-166 (2001)

44. Hohl, F., Mehrmann, L., Hamdan, A.: A context system for a mobile service platform. In: Schmeck, H., Ungerer, T., Wolf, L. (eds.) ARCS 2002. LNCS, vol. 2299, pp. 21-33. Springer, Heidelberg (2002)

45. Henricksen, K., Indulska, J., Rakotonirainy, A.: Modeling context information in pervasive computing systems. In: Mattern, F., Naghshineh, M. (eds.) PERVASIVE 2002. LNCS, vol. 2414, pp. 167-180. Springer, Heidelberg (2002)

46. Hightower, J., Brumitt, B., Borriello, G.: The location stack: A layered model for location in ubiquitous computing. In: Proceedings of the Fourth IEEE Workshop on Mobile Computing Systems and Applications (WMCSA 2002). IEEE Computer Society Press, Los Alamitos (2002)

47. Abowd, G.D.: Software engineering issues for ubiquitous computing. In: Proceedings of the 21st international conference on Software engineering, pp. 75-84. IEEE Computer Society Press, Los Alamitos (1999)

48. Patel-Schneider, P.F., Hayes, P., Horrocks, I. (eds.): OWL Web Ontology Language Semantics and Abstract Syntax. W3C Recommendation 10 February (2004), http://www.w3 . org/TR/owl-semantics 